\title{
Dissemination of Messages to Distant Vehicles in Sparse Condition via RSU as a Backbone
}

\author{
Jayanthi.S \\ Angel College of Engg. \\ and Technology \\ Tirupur, TamilNadu, \\ India
}

\author{
Rajeswari.M \\ Dept Of CSE \\ Angel College of Engg. \\ and Technology \\ Tirupur, TamilNadu, \\ India
}

\author{
Umamaheswari.P \\ Dept Of CSE \\ Info Institute Of \\ Technology \\ Coimbatore, \\ TamilNadu, India
}

Praveena. $R$

Angel College of Engg. and Technology

Tirupur, TamilNadu,

India

\author{
Aswiga.R.V \\ Angel College of Engg. and Tech. \\ Tirupur, TamilNadu, \\ India
}

\begin{abstract}
Vehicular Ad hoc NETworks (VANETs) are a variety of Mobile Ad hoc NETwork (MANET) that are used for communication among vehicles and between vehicles and road side equipments. For efficient communication, the routing protocols must be reliable and robust. In our work we deploy the RoadSide Units (RSUs) as an infrastructure and make use of it for the packet delivery. We carry and forward the message via RSU. In existing work they made use of RSU but that led to overhead and it is a time consuming, since they communicate as: source vehicle to its RSU, between RSUs and then from destination RSU to destination vehicle. But our work helps the user to query the destination RSU and receive the reply by requesting source RSU. And this source RSU communicates intermediate RSUs to communicate to the destination RSU and replies. Here the RSUs are connected. So, this helps in reducing the overhead and time taken. In addition our system provides the security to the user and the information transferred by producing Cryptographic MIXed key (CMIX).
\end{abstract}

Keywords: Vehicular Ad hoc NETworks (VANETs); RoadSide Units (RSUs); Reliable; Robust; Cryptographic mixed key (CMIX).

\section{INTRODUCTION}

The advances in the wireless technology paved way for the emergence of Vehicular Ad hoc NETworks (VANETs). As Mobile Ad hoc NETwork (MANET), VANET are also infrastructure less network but here the nodes participating are the vehicles. 
VANET allow vehicles to connect between them. Different from MANET, VANET allows vehicles to move in an organized manner, since the restriction of roads, buildings, etc. The vehicular communication is classified as two sections: Vehicle to Vehicle communication (V2V) and Vehicle to Infrastructure (V2I) or Vehicle to Roadside (V2R).

Another major difference of VANET from MANET is that the routing protocols of MANET are not suitable for VANET in most scenarios. This is because VANET are formed by moving vehicles, have high node mobility and limited in mobility patterns. The major division of VANET routing protocols are: topology based and geographic based routing protocols. Many protocols are proposed under these categories only.

The vehicles in the VANET are equipped with On Board Unit (OBU), Global Positioning System (GPS), digital maps, navigation system, etc. The RSUs are deployed in various places with its coverage area. These RSUs are in turn connected with internet.

Our work is mainly concentrated on routing packets efficiently. Many proposed work has its own measures to provide good performance. Here we deploy many RSUs and make use of them to disseminate packets to distant vehicles. This works well in both dense and sparse conditions. Most of the proposed protocols works well in dense condition were the participating vehicles are available at most of the time. Whereas in sparse condition the participating vehicle density is low. Our work is motivated to get rid of this environment. To make the system work well in sparse condition, we deploy the RSU at certain intervals with their coverage range.
The system works as follows: The source vehicle $\mathrm{S}$ needs to sends the packet to the destination vehicle $\mathrm{D}$. but the location of $\mathrm{D}$ is too far. At the time of routing, the density of vehicle is also less. So we make use of RSUs. The source vehicle s route the packet to its respective RSU. If there is available vehicle that directs to destination, then route the packet to this vehicle. Else the RSU disseminate to the neighboring RSU (may be destination vehicle's RSU) and from this intermediate RSU to other intermediate RSUs if needed or directs to the destination RSU. This RSU route the packet to the desired destination vehicle D.

Our work is different from the existing is: In existing work the source vehicle $S$ needs to send a packet to the destination vehicle $\mathrm{D}$ which is also too far. There are RSUs at various regions. The source packet is sent to the RSU within its coverage. This RSU seeks for a vehicle to carry this packet and founds any then pass the packet. If no vehicles found then the packets resides in the RSU. This intermediate vehicle carries the packet and delivers to its RSU and so on. When the packet reaches the destination RSU, it is delivered to the destination vehicle. Here at each stage of forwarding the vehicles are involved. There is no direct contact between RSUs. So here the involvement of the vehicle takes time because the RSU waits for the vehicle and the time taken by this vehicle to carry the packet to next RSU. Also produce the overhead at RSU since the packet resides there still the desired vehicle is found. 
So, our system gains the performance than the proposed and reduces the overhead and the time taken for forwarding.

The rest of this paper is designed as follows: the related work is depicted in section 2 . In section 3 the proposed work is described. Finally section 4 the work is concluded and the future work is discussed.

\section{RELATED WORK}

Y. Ding et. al. proposed to improve the delivery performance by deploying static nodes at intersection. In addition the adjacent nodes measure the delay of forwarding data between each other in real time, so that the routing decision can adapt to changing vehicle densities.

SADV reduces the data delivery delay through three mechanisms: SNAR (Static Node Assisted Routing): Here the intersection stores the path and forwards the best path.

LDU (Link Delay Update): Here the static nodes measure the link delay between each other in real time.

MPDD (MultiPath Data Delivery): It is used to decrease the packet delivery delay by trying to hit a faster delivery path.

C. Lochert et. al. proposed the Geographic Source Routing (GSR). This combines position based routing with the topological knowledge that is suited for city environments. Then they compared GSR with the other topology based approaches like DSR and AODV and conclude that GSR performs is enhanced than the other.
J. Zhao et. al. they concentrated on the problem of delay tolerant applications in the sparse network. This use the carry and forward method with the predicted mobility pattern. For this they projected some vehicleassisted data delivery (VADD) protocols: LVADD (Location First Probe), D-VADD (Direction First Probe), MD-VADD (Multipath Direction First Probe) and $\mathrm{H}$ VADD (Hybrid Probe). This can be selected based on the techniques used for road selection at the intersection.

R. Frank et. al. they used the TrafRoute routing protocol that involves selfelection and this self election is based on position, knowledge of the road topology and node density. Then this is suitable for both the V2V and V2I. This is very effective for short distance and to make use of the presence of infrastructure for longer distances.

R. Lu et. al. proposed the FLIP: An efficient privacy preserving protocol for finding like-minded vehicles on the road. This uses a security scheme Interest Privacy (IP). In this find the likeminded vehicles and secure sharing takes place via shared session key and distinguishes from those vehicles that are not likeminded.

\section{PROPOSED WORK}

In all above mentioned works they dint concentrate about the intersection issues. Because at the intersection the vehicle may take another route that never reach the destination vehicle. They just relied on moving vehicles to carry and forward packets. 
But in our work we deploy RSUs on road side and at intersection, when needed. This RSU stores the packet and deliver to intermediate vehicle or to intermediate RSU. If intermediate vehicle change their path, then with the help of RSU we can request that packet to be resend. But automatically the RSU resend the failed packets if it dint receive any acknowledgement. So this helps in efficient routing without dropping any packets.

Then the security which plays a major role in VANET. For security we use Cryptographic MIXed key (CMIX), were cryptographic keys are generated for the users. In VANET for the vehicle to participate in communication, it must be authenticated and get certified by Certification Authority (CA).

Once the CMIX are generated then they use only those keys to authenticate themselves to other users. Each RSU authenticates the vehicles under its coverage. Only after authenticated by RSU the vehicle can participate in the communication. So this enables the robust routing.

\subsection{Proposed Architecture}

The above described work is depicted by architecture diagram as in figure 1 . In the figure, there are vehicles that are moving. They are authenticated by RSU to participate in the network. The RSUs are connected to the server.

The source vehicle $\mathrm{S}$ needs to send a packet to the destination vehicle the arrow 2.D. But the location of D is extreme. So $\mathrm{S}$ route the packet to the RSU of its region. This is indicated by the arrow 1 . Next this RSU, communicate with the neighboring RSU and disseminate the packet. This RSU act as an intermediate node. This process is specified by the arrow 2 . Then this RSU send the packet to its neighboring RSU which is the destination vehicles' RSU. This process is noted by the arrow 3 . Finally from this RSU searches the destination vehicles' location and the packet is delivered to the destination vehicle. This process is notified by the arrow 4 .

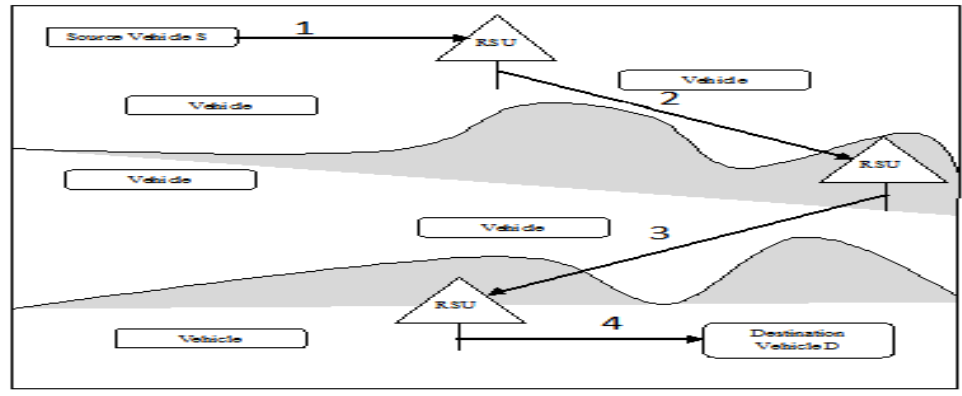


International Journal of Computer Applications Technology and Research Volume 2- Issue 5, 618 - 622, 2013, ISSN: 2319-8656

Figure 1. Proposed Architecture

\section{CONCLUSION}

In this paper we have projected the routing efficiency and robustness. The proposed work uses the infrastructure (RSU) as a backbone to route the packets to distant vehicles in sparse environment. This helps in reducing the load and complexity in transferring the data efficiently. And with this the security is ensured by CMIX that excludes the unauthorized vehicle.

\section{References}

[1]. R. Frank, E. Giordano, and M. Gerla, "TrafRoute: A different approach routing in vehicular networks," in Proc. VECON, Niagara Falls, ON, Canada, pp. 521-528, 2010.

[2]. J. Zhao and G. Cao, "VADD: Vehicleassisted data delivery in vehicular ad hoc networks," IEEE Trans. Veh. Technol., vol. 57, no. 3, pp. 1910-1922, May 2008.

[3]. Y. Ding, C. Wang, and L. Xiao, "A staticnode assisted adaptive routing protocol in vehicular networks," in Proc. VANET, New York, pp. 59-68, Sep. 2007.

[4]. C. Lochert, M. Mauve, H. Füÿler, and H. Hartenstein, "Geographic routing in city scenarios," SIGMOBILE, vol. 9, no. 1, pp. 6972, Jan. 2005.

[5]. R. Lu, X. Lin, X. Liang, and X. Shen, "FLIP: An efficient privacy preserving protocol for finding like-minded vehicles on the road," in Proc. Globecom, pp. 1-5, 2010. 